\title{
Gestational Age at Delivery and Doppler Waveforms in Very Preterm Intrauterine Growth-Restricted Fetuses as Predictors of Perinatal Mortality
}

Giancarlo Mari, MD, Farhan Hanif, MD, Marjorie C. Treadwell, MD, Michael Kruger, MA

Objective. The aim of this study was to compare gestational age at delivery and the performance of middle cerebral artery (MCA), ductus venosus (DV), and umbilical artery Doppler parameters in the prediction of perinatal mortality and morbidity in intrauterine growth-restricted (IUGR) fetuses delivered at 32 weeks or earlier. Methods. The study population consisted of 41 patients with IUGR fetuses. Delivery occurred for maternal or fetal indications. Two-tailed $\chi^{2}$ and Fisher exact tests, an independent $t$ test, and logistic regression were used for the analysis. $P<.05$ was considered statistically significant. Results. Gestational age at delivery ranged between 23.1 and 32 weeks (median, 27.6 weeks). There were 17 perinatal deaths. Ninety-four percent of the perinatal deaths occurred when the fetuses were delivered before 29 weeks. No fetus survived when delivered before 25 weeks. Two parameters predicted the perinatal mortality: gestational age at delivery (odds ratio, $0.52 ; 95 \%$ confidence interval, $0.31-0.88$ ) and the combination of abnormal MCA peak systolic velocity + DV reversed flow (odds ratio, 10.2; 95\% confidence interval, 1.8-57). For each week of pregnancy, there was a reduction in perinatal mortality of $48 \%$. No Doppler parameters were significantly associated with perinatal morbidity. Conclusions. Gestational age at delivery and the combination of abnormal MCA peak systolic velocity + DV reversed flow in very preterm IUGR fetuses were the best parameters in predicting perinatal mortality. The decreased perinatal mortality that is found for each week IUGR fetuses remain in utero should be taken into account when a decision to deliver an IUGR fetus before 30 weeks is made. Key words: biophysical profile; ductus venosus; middle cerebral artery; peak systolic velocity; pulsatility index.

\section{Abbreviations}

ARF, absent/reversed flow; $\mathrm{Cl}$, confidence interval; DV, ductus venosus; GA, gestational age; IUGR, intrauterine growth restriction; MCA, middle cerebral artery; PI, pulsatility index; PSV, peak systolic velocity; RF, reversed flow; UA, umbilical artery

Received February 5, 2007, from the Department of Obstetrics and Gynecology, Wayne State University, Detroit, Michigan USA (G.M., F.H., M.K.); and University of Michigan, Ann Arbor, Michigan USA (M.C.T.). Revision requested March 5, 2007. Revised manuscript accepted for publication March 19, 2007.

We thank the staff, technicians, nurses, and faculty of the Perinatology Research Branch and the Department of Obstetrics and Gynecology at Wayne State University for their support. This work was supported in part by the Intramural Research program of the National Institute of Child Health and Human Development, National Institutes of Health, Department of Health and Human Services.

Address correspondence to Giancarlo Mari, MD, Department of Obstetrics and Gynecology, Wayne State University, John R, Hutzel Hospital, 7 Brush, Detroit, MI 48201 USA.

E-mail: gmari@med.wayne.edu

Article includes CME test
I ntrauterine growth restriction (IUGR) has been defined as failure to attain optimal intrauterine growth. Although scientifically correct, this definition can is dificult to apply in practice because "optimal growth" cannot be easily determined. The American College of Obstetricians and Gynecologists has chosen to define IUGR as a fetus with an estimated weight below the 10th percentile for gestational age $(\mathrm{GA})^{1}$ because perinatal mortality and morbidity increase when the birth weight is below that percentile. ${ }^{2-4}$ With approximately 4 million births per year in the United States, 400,000 neonates will have a birth weight below the 10th percentile. Not all IUGR neonates are at risk for adverse outcome; some are constitutionally small but otherwise healthy neonates. ${ }^{5}$ 
Although several factors are implicated in the origin of IUGR, it has been suggested that the most important role appears to be played by placental insufficiency. ${ }^{6-8}$ Placental insufficiency causes increased placental vascular resistance, and it can be assessed by Doppler ultrasonography of the umbilical artery (UA). ${ }^{7,8}$

Intrauterine growth restriction secondary to placental insufficiency is a major cause of perinatal morbidity and mortality in the United States. ${ }^{9}$ Once IUGR is identified, obstetric management is focused on ensuring safety while the fetus continues to mature within a potentially hostile intrauterine environment. In the United States, the standard of care for management and delivery of the IUGR fetus at less than 32 weeks is based on serial biophysical profile evaluations or cardiotocography, ${ }^{10}$ whereas in Europe, it is based on the results of cardiotocography. ${ }^{11,12}$

Recently, it has been suggested that in IUGR fetuses, abnormal ductus venosus (DV) Doppler parameters are excellent predictors of adverse perinatal outcome. ${ }^{11-15}$ A parameter that has not been well investigated in IUGR fetuses is the middle cerebral artery (MCA) peak systolic velocity (PSV). One pilot study reported that the MCA PSV is increased in IUGR fetuses. ${ }^{16}$ More recently, we have reported that the MCA PSV predicts perinatal mortality better than the MCA pulsatility index (PI). ${ }^{17}$ This may be due to better correlation of blood velocity with blood flow compared with the PI (K. Clarke, PhD, oral communication, 2004).

The aim of this study was to compare GA at delivery and the performance of UA, MCA, and DV Doppler parameters in the prediction of perinatal mortality and morbidity in preterm severe IUGR fetuses.

\section{Materials and Methods}

\section{Clinical Procedures}

This was a retrospective study. The patients included in this study were either involved in research protocols approved by the Human Investigation Committee or part of a database on IUGR fetuses, and their inclusion for this study was approved by the Human Investigation Committee.

Among fetuses with an estimated weight below the third percentile and a UA PI below the 95\% confidence interval $(\mathrm{CI}),{ }^{18}$ we selected those without structural anomalies, delivered at 32 weeks' gestation or earlier, for whom Doppler measurements of the DV and MCA were obtained. The presence of absent/reversed flow (ARF) for the DV as well as the UA was also determined. Gestational age at delivery was based on certain last menstrual period or second-trimester ultrasonographic dating. Delivery was indicated in the presence of nonreassuring fetal testing, fetal death, or worsening maternal or fetal conditions as determined by the managing physician. Nonreassuring fetal testing was defined by the presence of either continuous variable/late decelerations or a biophysical profile of 4 or less. When delivery was indicated and the fetus was less than $500 \mathrm{~g}$, the patient was counseled on the poor prognosis and offered the option of nonintervention. Steroids were administered to all pregnant patients when fetuses were considered viable. Fetuses with abnormal karyotypes or suspicion of infection were not included in the study. Fetal weights were calculated and evaluated according to the nomograms established by Hadlock and coworkers. ${ }^{19}$

Pulsed wave Doppler ultrasonographic studies were performed with 1 of 4 color Doppler systems (Sequoia, Siemens Medical Solutions, Mountain View, CA; Voluson 530 and 730 Expert, GE Healthcare, Milwaukee, WI; and HDI 5000, Philips Medical Systems, Bothell, WA) using 3.5or $5-\mathrm{MHz}$ probes. All recordings were obtained in the absence of fetal breathing and fetal movements. For each vessel, an average of 3 consecutive Doppler velocity waveforms was used for statistical analysis. A free loop of the UA was sampled, and the PI was used to analyze the waveforms. The MCA was studied as reported previously, ${ }^{20}$ maintaining as close as possible to $0^{\circ}$ for the angle of insonation. The MCA waveforms were quantified by using the PI as well as the PSV. The DV was sampled soon after its origin from the umbilical vein.

The MCA PI was considered abnormal if the measurements were below the lower limit of normal reported previously ${ }^{20}$; the MCA PSV was considered abnormal if the measurements were above the upper limit of normal established previously. ${ }^{21}$ A UA PI above the $95 \%$ CI for GA of our reference range was considered abnormal. 
Absent or reversed end-diastolic velocity in the UA was also considered abnormal. For the DV, different patterns, abnormal DV $\mathrm{PI}^{22}$ and ARF in coincidence with atrial contraction, were considered independently as 2 different patterns of progressive severity. We also considered the combination of abnormal MCA PSV + DV reversed flow (RF).

Perinatal mortality was defined as fetal mortality occurring between 20 weeks' gestation and 28 days after birth. Major neonatal complications included grade III or IV intracerebral hemorrhage according to the classification of Papile et $\mathrm{al}^{23}$ and bronchopulmonary dysplasia.

\section{Statistical Analysis}

We evaluated the Doppler parameters (UA PI, UA ARF, UA RF, MCA PSV, MCA PI, DV PI, DV ARF, DV $\mathrm{RF}$, and the combination abnormal MCA PSV + DV RF) and the GA at delivery against the perinatal outcome. When a fetus had serial measurements performed, the last measurement before delivery was considered for the analysis.

The Doppler parameters were analyzed as categorical variables. The $\chi^{2}$ and Fisher exact tests were used for categorical variables. The significant parameters were further analyzed by a forward stepwise logistic regression analysis. In this analysis, we also included the GA. A $t$ test for independent groups was used to compare whether there was a difference in GA at delivery between the neonates who survived and those who died either in utero or within the first 4 weeks of life. $P<.05$ was considered to indicate statistical significance. Statistical analysis was performed with SPSS version 14.0 statistical software for Windows (SPSS Inc, Chicago, IL).

\section{Results}

Among the 41 fetuses who met the inclusion criteria, GA at delivery ranged between 23.1 and 32 weeks (median, 27.6 weeks). Twenty-nine fetuses were studied within 24 hours of delivery. The other 12 fetuses were studied within 72 hours of delivery. Characteristics and perinatal outcomes of the study population are reported in Table 1. Twenty-four women had no illnesses before the pregnancy. Where maternal illnesses before pregnancy existed, problems included chronic
Table 1. Characteristics of the Study Population $(N=41)$

\begin{tabular}{lll}
\hline Characteristic & \multicolumn{2}{c}{ Median (Range) } \\
\hline GA at Doppler study, wk & 27.6 & $(23-32)$ \\
Interval between last scan and delivery, d & 1 & $(0-3)$ \\
GA at delivery, wk & & \\
Total population & 27.6 & $(23.1-32)$ \\
Perinatal deaths $(n=17)$ & 26.6 & $(23.1-30.3)$ \\
Intrauterine fetal deaths $(n=7)$ & 26 & $(23.1-28.6)$ \\
Neonatal deaths $(n=10)$ & 26.8 & $(24.6-30.3)$ \\
Survivors $(n=24)$ & 29 & $(25.2-32)$ \\
Birth weight, $g$ & & \\
Total population & 550 & $(282-1240)$ \\
Perinatal deaths $(n=17)$ & 471 & $(282-680)$ \\
Intrauterine fetal deaths $(n=7)$ & 440 & $(282-680)$ \\
Neonatal deaths $(n=10)$ & 490 & $(300-660)$ \\
Survivors $(n=24)$ & 685 & $(360-1240)$ \\
\hline
\end{tabular}

hypertension ( $\mathrm{n}=15)$, asthma $(\mathrm{n}=1)$, and hyperthyroidism $(n=1)$. Indications for delivery were nonreassuring fetal testing $(n=21)$, severe preeclampsia $(n=8)$, HELLP (hemolytic anemia, elevated liver enzymes, low platelet count) syndrome $(n=5)$, and intrauterine fetal death $(n=7)$. All fetuses except those who died in utero were born by cesarean delivery.

The univariate analyses indicated that the following parameters were significantly associated with perinatal mortality: UA RF, abnormal MCA PSV, DV RF, and the combination of abnormal MCA PSV + DV RF (Table 2). When these parameters were entered in the logistic regression, it was found that 2 parameters predicted perinatal mortality: GA at delivery and the combination abnormal MCA PSV and DV RF (Nagerlke $R^{2}=55$; Table 3). Fetuses who died were at a significantly lower GA at the time of delivery than those who survived $(P<.05)$. For each additional week the fetuses remained in utero, perinatal mortality decreased by $48 \%$. One assumption that our study makes is that there is a linear relationship between GA at delivery and mortality. Although this might not be the case for all gestations, the relationship between these 2 variables can be considered linear to a first approximation at early GAs (eg, between 25 and 29 weeks).

Among the fetuses who survived, none had grade III or IV intraventricular hemorrhage. Bronchopulmonary dysplasia developed in 14 fetuses. No Doppler parameters were significantly associated with bronchopulmonary dysplasia. 
Table 2. Unadjusted Odds Ratios for Doppler Parameters Selected by the Univariate Analysis to Predict Perinatal Mortality $(N=41)$

\begin{tabular}{lcccc}
\hline & & \multicolumn{2}{c}{$\mathbf{9 5 \%} \mathbf{C l}$} & \\
\cline { 3 - 4 } Parameter & OR & Lower & Upper & $P$ \\
\hline MCA PI & 0.3 & 0.07 & 1.2 & .15 \\
UA ARF & 0.8 & 0.6 & 1.0 & .06 \\
DV PI & 2.8 & 0.8 & 10.5 & .2 \\
DV RF & 9.1 & 2.1 & 38.3 & .003 \\
MCA PSV & 11.4 & 1.3 & 101 & .01 \\
MCA PSV + DV RF & 12 & 2.7 & 53.6 & .001 \\
UA RF & 13.5 & 1.5 & 119 & .006 \\
\hline
\end{tabular}

OR indicates odds ratio.

\section{Discussion}

Our study showed that the GA at delivery and the combination of abnormal MCA PSV + DV RF were the best predictors of perinatal mortality in IUGR fetuses delivered earlier than 32 weeks. Our data also showed that there was a $48 \%$ reduction in the incidence of perinatal mortality for every additional week of gestation at delivery. Although we included fetuses with an estimated fetal weight below the third percentile, we believe that out data can be applied to fetuses with an estimated weight below the 10th percentile who have abnormal UA Doppler parameters.

Previous studies have emphasized the appearance of a temporal sequence of Doppler and biophysical changes that precede the peripheral and central circulatory system changes of the severely growth-restricted fetus. ${ }^{11-13}$ It has been suggested that an abnormal DV has the potential to become a key factor in the management and optimum timing for delivering the IUGR fetus. Indeed, some investigators perform a cesarean delivery simply on the basis of the deterioration of the DV Doppler waveforms. ${ }^{24}$

Table 3. Parameters Selected by the Forward Stepwise Logistic Regression Analysis as Best Predictors of Perinatal Mortality in 41 IUGR Fetuses

\begin{tabular}{lcccc}
\hline & & \multicolumn{2}{c}{$95 \% \mathrm{Cl}$} & \\
\cline { 3 - 5 } Parameter & OR & Lower & Upper & $\boldsymbol{P}$ \\
\hline MCA PSV + DV RF & 10.2 & 1.8 & 57 & .008 \\
GA at delivery & 0.52 & 0.31 & 0.88 & .01 \\
\hline
\end{tabular}

OR indicates odds ratio.
Although the blood flow changes seen in the DV are important predictors of a poor outcome, the mechanisms to determine the optimal timing of IUGR fetus delivery before 32 weeks remain controversial. It would be very important to determine whether delivery of the IUGR fetus at less than 32 weeks should be based only on changes in the DV with identification of ARF in this vessel or whether delivery should be based rather on other testing parameters. The overwhelming impact of GA on survival suggests that additional information about the IUGR fetus at less than 32 weeks might potentially allow us to delay delivery in selected cases and improve the outcome. Therefore, further evaluation of additional measurements, including the MCA PSV, and acknowledgment of various methods of fetal testing, including cardiotocography and biophysical profile changes, are crucial to understanding how to optimize obstetric care in this high-risk population.

We have previously determined that the MCA PSV increased after the decreased MCA PI and remained above its normal reference range up to a few hours before a fetal death or nonreassuring fetal testing occurred. ${ }^{17}$ We believe that it is not necessary to assess the MCA PSV and DV in IUGR fetuses if there is forward diastolic flow in the UA. This initial and qualitative analysis of the UA (present versus absent end-diastolic velocity) could be performed by most sonographers. When the UA end-diastolic velocity is absent, the fetus could be referred for more sophisticated tests at appropriate centers.

In our study, $94 \%(16 / 17)$ of the perinatal mortality occurred when the fetuses were delivered before 29 weeks. No fetus survived when delivered at a GA earlier than 25 weeks. It must be emphasized that IUGR fetuses who are detected early in the third trimester have a significant decrease in perinatal mortality for each week that they remain in utero; this should be taken into account when a decision to deliver an IUGR fetus before 30 weeks is made.

Some weaknesses of our study included the fact that the study was not longitudinal; therefore, we were not able to determine when serial changes occurred in both the MCA PSV and DV blood velocity. The IUGR group reported here represented a heterogeneous group because maternal 
conditions present before pregnancy and those occurring during pregnancy influenced the time of delivery in many of our fetuses. Moreover, the small number of patients may have limited the ability of individual Doppler parameters to predict perinatal morbidity.

A longitudinal study of DV and MCA PSV that includes a larger number of fetuses, considers long-term outcome, and also differentiates between idiopathic IUGR fetuses on the one hand and IUGR fetuses associated with known causes of placental insufficiency on the other is warranted at this time.

\section{References}

1. American College of Obstetricians and Gynecologists. ACOG Practice Bulletin: Intrauterine Growth Restriction. Number 12. Washington, DC: American College of Obstetricians and Gynecologists; 2000.

2. Garite TJ, Clark R, Thorp JA. Intrauterine growth restriction increases morbidity and mortality among premature neonates. Am J Obstet Gynecol 2004; 191:481-487.

3. Lubchenco LO, Hansman C, Dressler M, Boyd E. Intrauterine growth as estimated from liveborn birthweight data at 24 to 42 weeks' gestation. Pediatrics 1963; $32: 793-800$

4. Manning FA. Intrauterine growth retardation. In: Manning FA (ed). Fetal Medicine: Principles and Practice. Norwalk, CT: Appleton \& Lange; 1995:307-393.

5. Fretts RC, Boyd ME, Usher RH, Usher HA. The changing pattern of fetal death, 1961-1988. Obstet Gynecol 1992; 79:35-39.

6. Brosens I, Dixon HG, Robertson WB. Fetal growth retardation and the arteries of the placental bed. $\mathrm{Br} J$ Obstet Gynaecol 1977; 84:656-663.

7. Karsdorp VH, van Vugt JM, van Geijn HP, et al. Clinical significance of absent or reversed end diastolic velocity waveforms in umbilical artery. Lancet 1994; 344:1664-1668.

8. Salafia CM, Pezzullo JC, Minior VK, Divon MY. Placental pathology of absent and reversed end-diastolic flow in growth-restricted fetuses. Obstet Gynecol 1997; 90:830836.

9. Matthews TJ, Menacker F, MacDorman MF. Infant mortality statistics from the 2001 period linked birth/infant death data set. In: National Vital Statistics Reports. Hyattsville, MD: National Center for Health Statistics; 2003;52.

10. American College of Obstetricians and Gynecologists. ACOG practice bulletin: antepartum fetal surveillance. Number 9, October 1999. Int J Gynaecol Obstet 2000; 68: 175-185.

11. Ferrazzi E, Bozzo M, Rigano S, et al. Temporal sequence of abnormal Doppler changes in the peripheral and central circulatory systems of the severely growth-restricted fetus. Ultrasound Obstet Gynecol 2002; 19:140-146.

12. Hecher $\mathrm{K}$, Bilardo $\mathrm{CM}$, Stigter $\mathrm{RH}$, et al. Monitoring of fetuses with intrauterine growth restriction: a longitudinal study. Ultrasound Obstet Gynecol 2001; 18:564-570.

13. Baschat AA, Gembruch $U$, Harman CR. The sequence of changes in Doppler and biophysical parameters as severe fetal growth restriction worsens. Ultrasound Obstet Gynecol 2001; 18:571-577.

14. Bilardo CM, Wolf H, Stigter RH, et al. Relationship between monitoring parameters and perinatal outcome in severe, early intrauterine growth restriction. Ultrasound Obstet Gynecol 2004; 23:119-125.

15. Cosmi E, Ambrosini G, D'Antona D, Saccardi C, Mari G. Doppler, cardiotocography, and biophysical profile changes in growth-restricted fetuses. Obstet Gynecol 2005; 106:1240-1245.

16. Ozcan T, Sbracia M, d'Ancona RL, Copel JA, Mari G. Arterial and venous Doppler velocimetry in the severely growth-restricted fetus and associations with adverse perinatal outcome. Ultrasound Obstet Gynecol 1998; 12 : 39-44.

17. Mari G, Hanif F, Kruger M, Cosmi E, Santolaya-Forgas J, Treadwell M. Middle cerebral artery peak systolic velocity: a new Doppler parameter in the assessment of growth restricted fetuses. Ultrasound Obstet Gynecol 2007; 29:310-316.

18. Gosling RG, King DH. Ultrasound Angiology. New York: Churchill-Livingstone; 1975:61-98.

19. Hadlock FP, Harrist RB, Martinez-Poyer J. In utero analysis of fetal growth: a sonographic weight standard. Radiology 1991; 181:129-133.

20. Mari G, Deter RL. Middle cerebral artery flow velocity waveforms in normal and small-for-gestational-age fetuses. Am J Obstet Gynecol 1992; 166:1262-1270.

21. Mari G, Adrignolo A, Abuhamad A, et al. Diagnosis of fetal anemia with Doppler ultrasound in the pregnancy complicated by maternal blood group immunization. Ultrasound Obstet Gynecol 1995; 5:400-405.

22. Hecher K, Campbell S, Snijders R, Nicolaides K. Reference ranges for fetal venous and atrioventricular blood flow parameters. Ultrasound Obstet Gynecol 1994; 4:381-390.

23. Papile LA, Burstein J, Burstein R, Koffler $\mathrm{H}$. Incidence and evolution of subependymal and intraventricular hemorrhage: a study of infants with birth weight less than 1,500 gm. J Pediatr 1978; 92:529-534.

24. Schwarze A, Gembruch U, Krapp M, Katalinic A, Germer U, Axt-Fliedner R. Qualitative venous Doppler flow waveform analysis in preterm intrauterine growth-restricted fetuses with ARED flow in the umbilical artery: correlation with short-term outcome. Ultrasound Obstet Gynecol 2005; 25:573-579. 